\title{
An automated Approach to Digitise Railway Bridges
}

\author{
M. Al-Adhami ${ }^{a}$, S. Rooble ${ }^{b}$, S. Wu ${ }^{c}$, C. Osuna-Yevenes ${ }^{b}$, V. Ruby-Lewis ${ }^{b}$, M. Greatrix ${ }^{b}$, \\ Y. Cartagena ${ }^{a}$, and S. Talebi \\ ${ }^{a}$ School of Art, Design and Architecture, University of Huddersfield, UK \\ ${ }^{b}$ Waldeck consulting, UK \\ ${ }^{c}$ School of Architecture and Built Environment, Nottingham Trent University \\ E-mail: mus.adhami@hud.ac.uk, sagal.rooble@waldeckconsulting.com, song.wu@ntu.ac.uk, clara.osuna- \\ yevenes@waldeckconsulting.com, veronica.ruby-lewis@waldeckconsulting.com, \\ mark.greatrix@waldeckconsulting.com, yreilyn.cartagenadelgado@hud.ac.uk, S.Talebi@hud.ac.uk
}

\begin{abstract}
-
In the $U K$, the railing sector has a large number of ageing masonry bridges that need to be examined every year. The traditional inspection regime consists of visual observation with manually recording the defect information observed on the structure. Previous studies have shown that this method of inspection is not reliable, time-consuming, depends on the inspector's judgment and result in further costs to the inspection process. Thus, there is a need to revolutionise the current inspection regime and improve the efficiency and reliability of the acquired data.

The development of digital surveying technology such as terrestrial laser scanning (TLS) and progressions in innovative software techniques will support the production of accurate representation of any asset, this automation, which results in achieving a tangible survey of a physical asset in a digital environment. In this paper, a strategic approach for an effective solution to automating the traditional process of surveying and processing point cloud data is presented, with further automation of implementing an automated bridge generation approach to large and complex cloud data sets. The presented digital method will generate a digital asset from the point cloud, and this will support surveyors and engineers to identify defects through various digital means. The presented approach has been tested on real-life projects which have demonstrated time-saving workflow on generating digital assets. This research will demonstrate the effective automation of point cloud to producing a physical asset of a structure with the ability to produce BIM model.
\end{abstract}

\section{Keywords -}

Bridge automation; Inspection; Masonry bridge; Terrestrial scanning; Point clouds; Scan to BIM

\section{Introduction}

In the UK, infrastructure management is facing significant challenges due to ageing assets and increasing demand. In the railway sector, it is estimated that there are approximately 29000 bridges, 22000 retaining walls, 21000 culverts, 200 miles of coastal defence and 600 tunnels, which require regular examination. The current visual examination process involves deploying a large workforce onto unsafe sites to inspect and report upon the status of each bridge on the network. There is increasing demand to replace the current cumbersome process (resource-intensive, unsafe conditions, sub-optimal process) with a faster, safer, digitally-enabled process using digital operators for mostly off-site inspection, automatic defects detection supported by an automated process, such as machine learning.

Due to the ageing nature of the assets, the original design information is often not readily available. The immediate challenge of implementing the digital process is to reconstruct the 3D BIM model from the captured data on-site. This paper aims to address this challenge by proposing a practical solution to rapid reconstruct the 3D BIM model from the point cloud data captured by reality capture technology. A real-life project is presented to demonstrate the effectiveness of the solution.

\section{Literature review}

\subsection{Reality capture technology}

Reality capture technology for recording the state of the existing structure has been a focus of many studies $[1$, 2]. Several technologies have been used for digital surveying such as terrestrial laser scanning (TLS), Terrestrial video and photogrammetry technology, and Unmanned Aerial Vehicle (UAV). The precision of these technologies is varied, which make some of them better than others on different surveying scenarios. For example, 
UAV is useful in post-disaster assessment, such as postearthquake evaluation [3], tornado and hurricanes damage assessment [4], that could give a general damage identification of a structure as a whole [5].

TLS can provide detailed information about the structure with higher accuracy than photogrammetry and UAVs [6]. Zeibak-Shini et al. [6] claimed that the TLS was applicable for damage assessment on building element with better quality than the UAV scanning. In Spain, different digital surveying technologies were used to document the as-is state of heritage masonry arch bridge, static and mobile TLS integrated with a digital camera were able to provide high accuracy $3 \mathrm{~d}$ digital model [7]. In the US, a study used pre- and post-event approach for damage assessment of buildings after a tornado, both aerial and TLS surveying technology were used to document the state of each building before and after the event to evaluate the damage caused to buildings [8]. In the same study, they have also estimated wind speed and path direction of the tornado using the same digital data.

TLS, terrestrial video, photogrammetry, and UAV can generate point clouds model of a scene, and other low-cost approaches have also been studied to generate the similar dataset such as digital photogrammetry technique called Structure-from-Motion (SfM) [9], Multi-View Stereopsis (MVS) [10], and the combination of both [11]. Even though the above techniques are able to reconstruct the surveyed structure in a $3 \mathrm{D}$ environment, none of them can automatically produce and recognise the structure at the component level that is required for a BIM model.

\subsection{Scan to BIM $-3 D$ reconstruction of BIM model}

Scan to BIM is also known as Point cloud to BIM, the process aims to use point cloud data to generate BIM model [12]. Typically, the point cloud data is obtained by TLS, and the data is transferred to BIM tools such as Revit, Bentley, Graphisoft, to reconstruct a BIM model. The problem of reconstruction of the as-as infrastructure/building assets is not a new area of research $[1,2]$. Currently, the scan to BIM process remains largely a manual process which is timeconsuming and error-prone [3]. In recent years, researchers around the world have been investigating ways to automate the process, including automatic identification of objects from the geometric features [13].

Roberts et al. [14] developed a technique to digitally capture a physical space from various points using the data to create an intelligent $3 \mathrm{D}$ model. The process provides a vital starting point for design teams that use BIM to iterate their designs and then manage, coordinate, and share all of the project information.

In buildings, the reconstruction of interiors and exteriors using the laser scanner was the case of many studies [4-6]. Although many algorithms presented for model reconstruction, most of them emphasise on model creation for visualisation rather than the accuracy of the geometries. Furthermore, the algorithms do not recognise the identity of components such as floors, walls, and ceilings [7]. Macher et al. [3] presented a semi-automatic approach, and segmentation was performed, so that point clouds corresponding to grounds, ceilings, and walls can be established. The output of building components can then be integrated into BIM software using IFC format. In the outdoor environment, Bassier et al. [8] used random forests classifier to automatically identify structural elements such as floors, ceilings, roofs, walls, and beams.

In the infrastructure management sector, several algorithms for $3 \mathrm{D}$ data processing and modelling have been developed to achieve surveying goals [9]. One of the significant studies was presented by Sacks et al. [10], they developed a $3 \mathrm{D}$ engine to create a geometric model of the assets associated with the point cloud model. The engine was based on surface primitive extraction algorithm, component detection, and classification algorithm for detection and classification. A set of training data was also presented to establish the relationships between surface primitives and the integrated component. However, the BIM reconstruction process is still at its infancy in geometrical creation, particularly for non-standard components with complex geometry.

\section{Bridge Scan to BIM approach}

In recent years, many algorithms have been developed to extract planar patches from point data or to recognise and identify the components of a structure such as walls, ceilings, and floors [15]. However, very few studies have successfully demonstrated the practicality of rapid reconstructing bridges from point cloud data in a large scale. In this paper, the authors propose a component library based approach especially developed for masonry railway bridges.

The result of the approach is a rich BIM model generation tool that is capable of producing Autodesk Revit BIM model with all the required information about each component constituent of a bridge structure. Furthermore, the proposed system follows the end-user requirements for condition assessment of railway bridge structure to ensure the resulting BIM model is semantically rich and suitable for condition assessment.

In this paper, three key aspects have been considered during the development of the system.

1. From a practical point of view, it is essential to have both data from the survey and BIM in the same development environment, not only for BIM model 
creation but also for component check and validation.

2. Clear definition of the relationships between the elements [16], i.e. bridge elementsneed to be connected in a logical format.

3. The output BIM model must comply with the international standard such as IFC schema.

The railway bridges have a wide variety of components in their geometrical shape, parameters, and position. For example, each bridge component from the same type could have different geometries, parameters, and positions within the overall structure, see Table 1.

Table 1. Different type of wing wall and connection to abutment

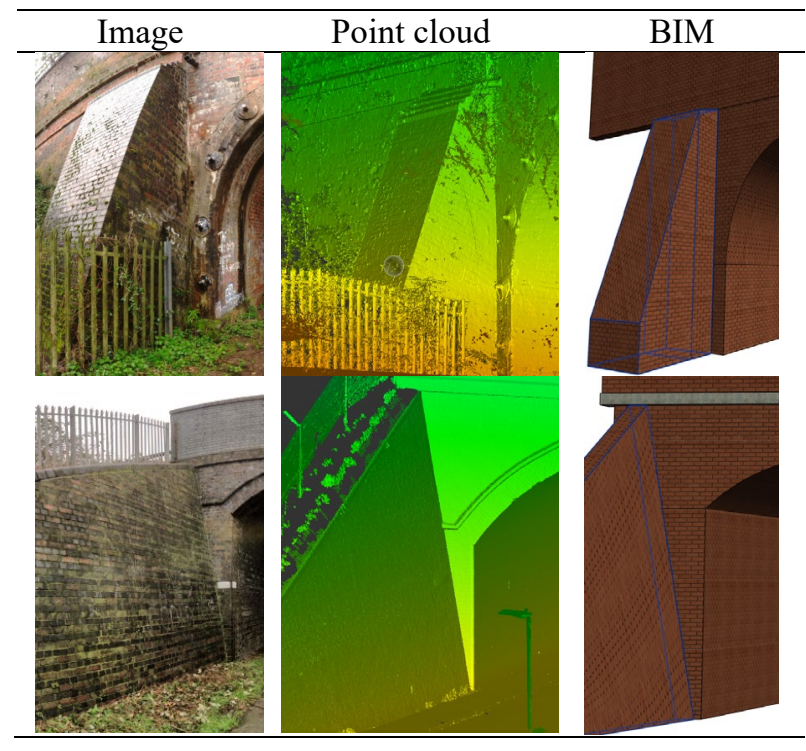

During the development, the authors recognise the relationships exists between the components in terms of the geometries, parameters and composition. As a result, a unique bridge component library is proposed to include all components and structural elements for bridges in order to reconstruct a bridge from point cloud data. A typical bridge can consist of several major components, such as Deck (DK), End Support (ES), and Intermediate supports (IS). Each major component is formed by several elements, such as Abutment, Wing wall, etc.

The bridge components and elements in the library are modelled based on the following protocols a) a logic of component reconstruction sequence, b) a number of required parameters for flexible geometries c) and relationships between components and elements. Figure 1 provides a high-level overview of the approach, which breaks the BIM reconstruction process into four stages.

In the first stage, a bridge component library is developed, and the bridge components and elements are categorised based on bridge condition marking index (BCMI) guideline. The BCMI helps develop a robust and flexible bridge component from a geometrical perspective that can be used in most bridges with a similar structure. The component library can be developed in standard BIM tools such as Autodesk Revit.

The second stage is to identify the component from the point cloud scene. It will examine if there is a new element, feature with the element, that is missing and/or not included in the library. This step is essential to maintain the library and keep it up to date with all components.

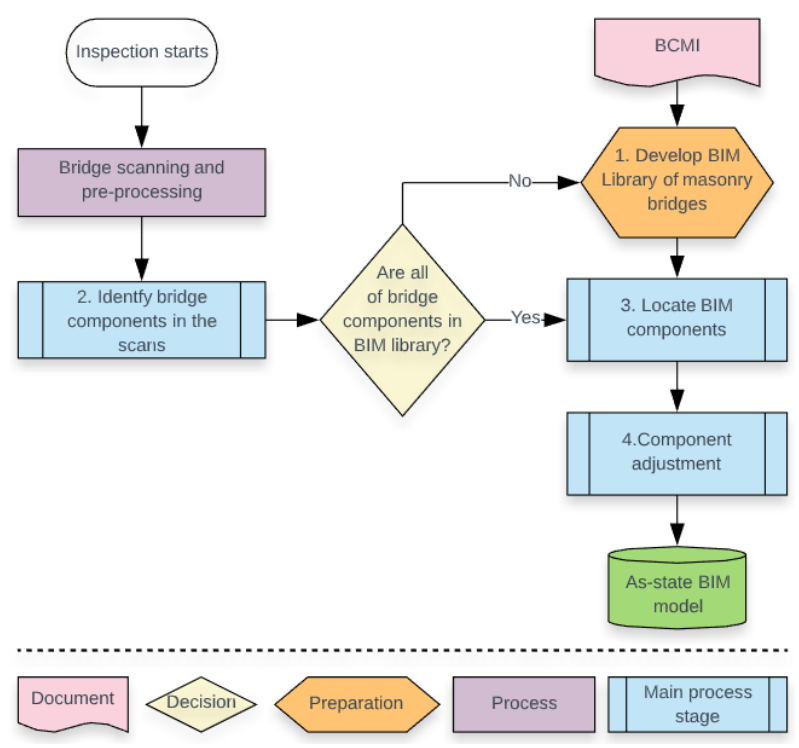

Figure 1. system overview

The third stage is to establish the position and orientation of individual components and elements of the bridge. The scanning provides a point cloud scene registered within a local coordinate system. The point cloud is then used to identify the spatial location of each bridge component. Moreover, the component's connection to other components in the whole structure can also be established based on the relationship rules in the library.

In the fourth stage, the bridge components are being reconstructed from elements in the library based on the information for previous stages. The parametric nature of each component and element gives the flexibility to adjust the geometries of the components based on the point cloud data. The algorithm will extract the required parameters of the individual components from the point cloud data. For the actual implementation, a set of algorithms is developed to connect all stages and interact with point cloud data and the component library.

The proposed approach has been tested on thirty railway bridges in the UK. In the following section, a brief description of the implementation on a single-span arch bridge is presented. 


\section{Implementation}

The single-span arch bridge consists of three major components, Deck (DK), End Support (ES), and Intermediate supports (IS). Figure 2 shows the structural system of the single-span masonry arch bridge. Major and minor elements, names, and codes are explained in Table 2.

The process of data acquisition, BIM reconstruction, and the validation of the produced model are explained in the following sections.

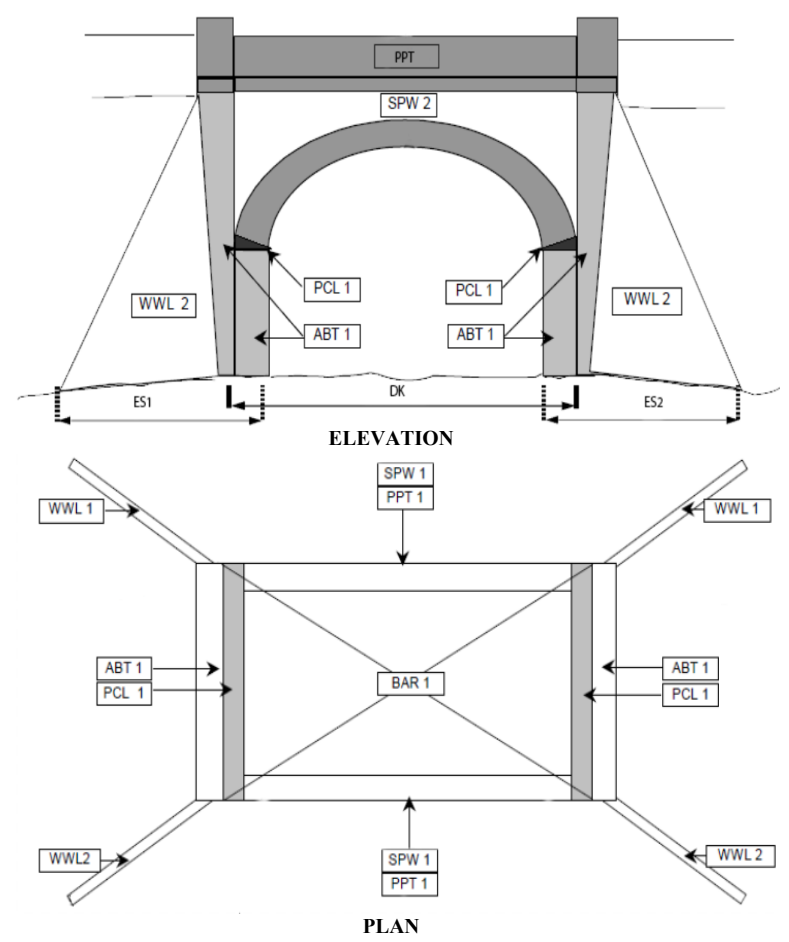

Figure 2. Single-span masonry arch bridge drawing

Table 2 elements in the single-span masonry arch bridge

\begin{tabular}{cc}
\hline Elements & Code \\
\hline Abutment & ABT \\
Wing wall & WWL \\
Barrel arch & BAR \\
Spandrel wall & SPW \\
Parapet & PPT \\
Padstone & PCL \\
\hline
\end{tabular}

\subsection{Data acquisition}

For the single-span masonry arch bridge as shown in Figure 3, TLS is used for data acquisition. The site condition, weather condition, and other environmental conditions such as site access, traffic situation, vegetation, flooding, etc, are assessed before the scan. The scanning set-points are also planned in order to cover the entire structure.

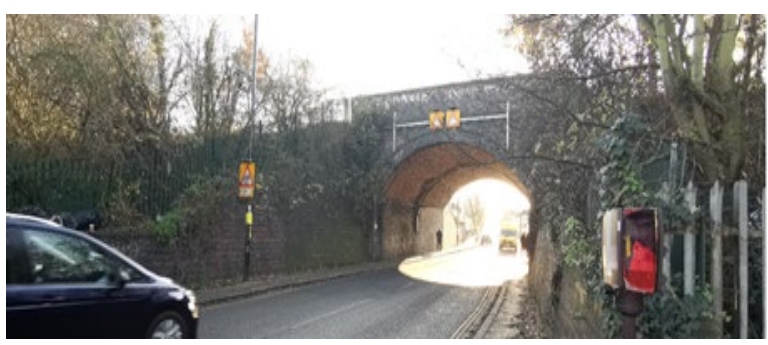

Figure 3. Single-span masonry arch bridge

On-site, the survey team sets up a ground control point (GCP) for the project. A Global Navigation Satellite System (GNSS) is used to record the GCP in the Ordnance Survey National Grid (OSGB) reference system, and the resulting coordinates will be used in the processing of TLS data sets. The TLS scan is performed at every pre-planned set-point to capture $3 \mathrm{D}$ point cloud. The individual scans are then registered to each other using automated software to produce a dense point cloud model of the bridge.

\subsection{BIM Reconstruction}

In the proposed system, the BIM reconstruction algorithm process the point cloud data and generate a BIM model almost automatically. Moreover, the proposed system does not require a clean point cloud model (noise points such as people, trees in the scene, are not removed). However, a clean model can save processing time and produce better results. In the singlespan masonry arch bridge, the scan by TLS has around 440million points (Figure 4).

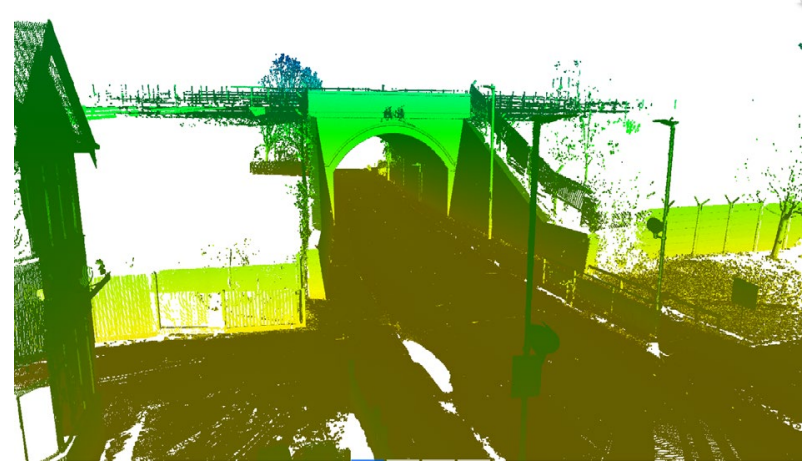

Figure 4. The point cloud model of the bridge

The resulting 3D BIM model as shown in Figure 5 is a digital representation of the bridge which is formed by the components and the corresponding structural elements of a masonry arch bridge listed in Table 2 .

The algorithm is able to identify the structural elements from the bridge point cloud scene. It assembles 
bridge components of the elements associated with the point cloud of the structure from the component library. It also fetches the required parameters of each component to finetune the BIM component geometry to fit the point cloud.

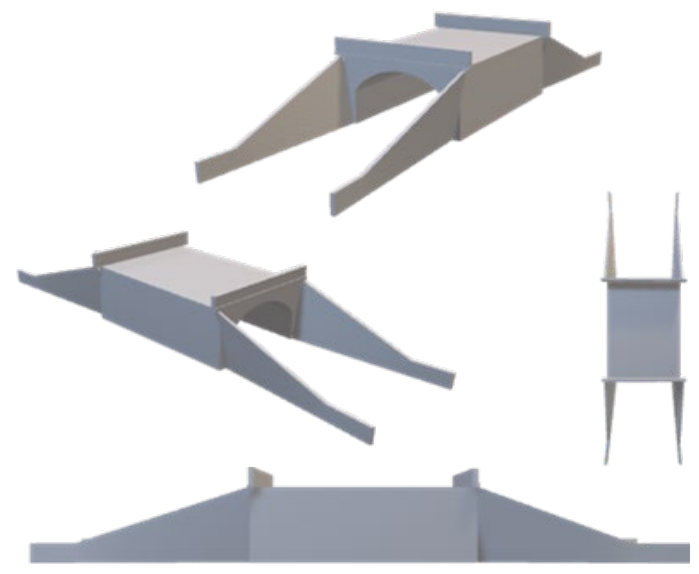

Figure 5. BIM model of the single-span arch bridge.

The algorithm would also position the bridge components following the density of the point cloud data and the relationships between components as shown in Figure 6
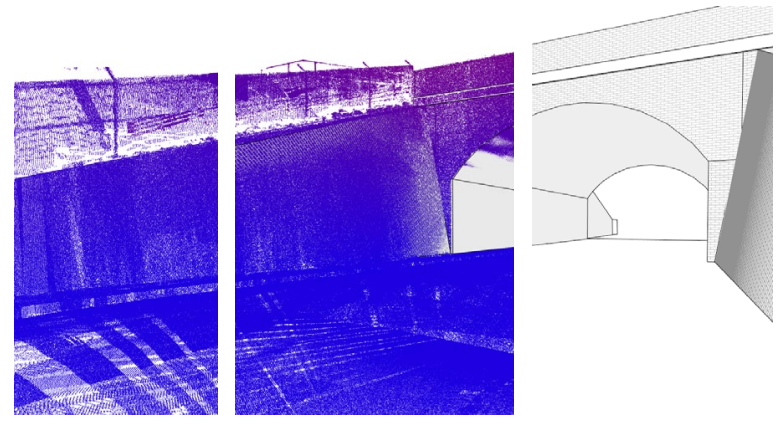

Figure 6 . bridge reconstruction

\subsection{Validation process}

The output BIM model is in Autodesk Revit format that can be exported in IFC. The BIM model is validated in Verity, a construction verification software. Verity would check the point cloud against each bridge component. The visual verification reports show if the structural components are generated correctly within the required $50 \mathrm{~mm}$ tolerance.

Figure 8 shows the BIM model of a Wing Wall (WWL) that is compared to the surveyed point cloud data under $50 \mathrm{~mm}$ tolerance. The colours in the model represent the distance of the points to the BIM component; green shows the perfect match while red is out of tolerance.
Figure 7 shows the results of bridge spandrel (SPW) to $50 \mathrm{~mm}$ tolerance. Although the SPW component passed the verification process, the result shows the point cloud does not fully match the BIM components. This could be because the BIM component is not flexible enough to fit the geometry of the bridge, or the point cloud model has too many noises that affect the verification process or both.

As previously mentioned, the system was validated with thirty bridges. Majority of the BIM models could meet the tolerance requirement. On occasion, the component would not line up with point cloud perfectly, and further adjustments are made to improve the accuracy of the bridge model.

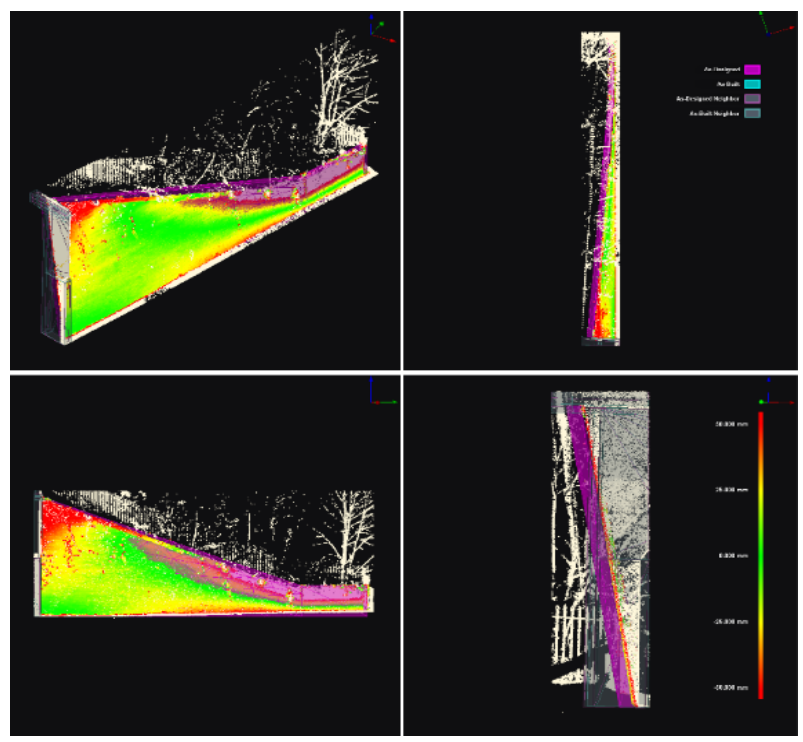

Figure 7. Verification results of the WWL

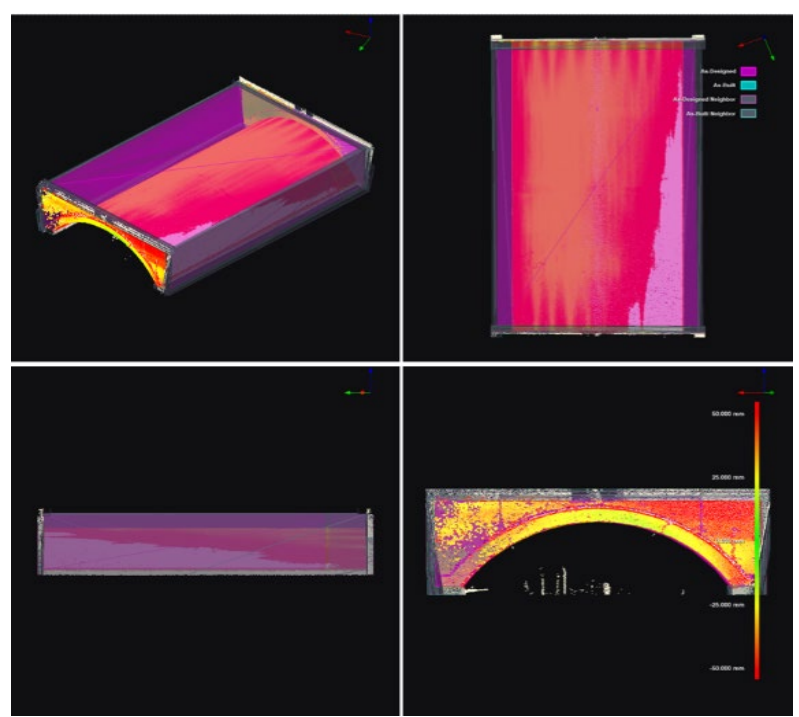

Figure 8. Verification results of SPW 


\section{Conclusion}

Reality capture technology is a promising solution for bridge condition assessment. However, the data acquired from scanning tools can not be directly used to establish a digital information system. Furthermore, the conventional process of creating a BIM model from point cloud data is a time-consuming manual process.

Due to the increasing demand for digitising UK infrastructure, there is a need to develop a workflow to rapid scan the asset and generate a BIM model for condition assessment. This paper proposed a new approach to automate the reconstruction of a BIM model of railway bridge from point cloud data, which can also be applied to other physical assets. The approach is demonstrated on an industry project of thirty bridges of different types, shapes, and spans.

The process includes capturing the masonry structure using TLS and producing a point cloud representation of the bridge and further generating a 3D BIM model. The output result of each bridge component is validated against the point cloud under $50 \mathrm{~mm}$ tolerance. The new process has reduced the traditional modelling times from days/weeks to hours for the thirty bridges. The project also identified several advantages and limitations of the proposed approach.

\section{Advantages}

- The system can process a complex structure of masonry bridges.

- The digital representation is sufficiently accurate for condition assessment.

- The algorithms work well with an uncleaned point cloud data and are still able to to generate an accurate BIM model.

- The system is very efficient compared to the manual process. (average $15 \mathrm{~min}$ processing time as opposed to hours in the manual process).

Limitations

- In some cases, it might require manual adjustment of the output in order to achieve $50 \mathrm{~mm}$ tolerance level.

- Further development of the library is required when dealing with a new type of bridge.

- The additional parameters of the components/element might be needed to accommodate a certain type of bridge.

Overall, this paper presented a new component library based scan to BIM approach for bridges. It has been tested on a real-life project with a significant number of bridges. Although the current library is limited to railway bridges, it is possible in the future to expand into other infrastructure assets, such as tunnels, culverts and retaining walls.

\section{References}

[1] Abu Dabous, S. and S. Feroz, Condition monitoring of bridges with non-contact testing technologies. Automation in Construction, 2020. 116: p. 103224.

[2] Koch, C., et al., Machine vision techniques for condition assessment of civil infrastructure, in Advances in Computer Vision and Pattern Recognition. 2015, Springer-Verlag London Ltd. p. 351-375.

[3] Liu, W., et al., Evaluation of three-dimensional shape signatures for automated assessment of postearthquake building damage. Earthquake Spectra, 2013. 29(3): p. 897-910.

[4] Mao, Z., et al. Towards Automated Post-Disaster Damage Assessment of Critical Infrastructure with Small Unmanned Aircraft Systems. 2018. Institute of Electrical and Electronics Engineers Inc.

[5] Dong, P. and H. Guo, A framework for automated assessment of post-earthquake building damage using geospatial data. International Journal of Remote Sensing, 2012. 33(1): p. 81-100.

[6] Zeibak-Shini, R., et al., Towards generation of asdamaged BIM models using laser-scanning and asbuilt BIM: First estimate of as-damaged locations of reinforced concrete frame members in masonry infill structures. Advanced Engineering Informatics, 2016. 30(3): p. 312-326.

[7] Puente, I., et al., NDT documentation and evaluation of the roman bridge of lugo using GPR and mobile and static LiDAR. Journal of Performance of Constructed Facilities, 2015. 29(1).

[8] Kashani, A.G., et al., Automated tornado damage assessment and wind speed estimation based on terrestrial laser scanning. Journal of Computing in Civil Engineering, 2015. 29(3).

[9] Westoby, M.J., et al., 'Structure-from-Motion' photogrammetry: A low-cost, effective tool for geoscience applications. Geomorphology, 2012. 179: p. 300-314.

[10] Furukawa, Y. and J. Ponce, Accurate, Dense, and Robust Multiview Stereopsis. IEEE Transactions on Pattern Analysis and Machine Intelligence, 2010. 32(8): p. 1362-1376.

[11] James, M.R. and S. Robson, Straightforward reconstruction of $3 D$ surfaces and topography with a camera: Accuracy and geoscience application. Journal of Geophysical Research: Earth Surface, 2012. 117(F3).

[12] Bosché, F., et al., The value of integrating Scan-to- 
BIM and Scan-vs-BIM techniques for construction monitoring using laser scanning and BIM: The case of cylindrical MEP components. Automation in Construction, 2015. 49: p. 201-213.

[13] Wang, Q., J. Guo, and M.-k. Kim, An Application Oriented Scan-to-BIM Framework. Remote Sensing, 2019. 11: p. 365.

[14] Roberts, C., et al., Digitalising asset management: concomitant benefits and persistent challenges. International Journal of Building Pathology and Adaptation, 2018. 36: p. 152-173.

[15] Xiong, X., et al., Automatic creation of semantically rich $3 D$ building models from laser scanner data. Automation in Construction, 2013. 31: p. 325-337.

[16]Borin, P. and F. Cavazzini, CONDITION ASSESSMENT OF RC BRIDGES. INTEGRATING MACHINE LEARNING, PHOTOGRAMMETRY $A N D$ BIM. ISPRS - International Archives of the Photogrammetry, Remote Sensing and Spatial Information Sciences, 2019. XLII-2/W15: p. 201208. 